\title{
Experiment Study of Outburst Pulverized Coal-Gas Two-Phase Flow and Characteristic Analysis of Outburst Wave
}

\author{
Xusheng Zhao, ${ }^{1,2}$ Jie Cao $\mathbb{D}^{1,2}$ Bo Wang $\mathbb{D}^{1,2}$ and Xuelin Yang ${ }^{1,3}$ \\ ${ }^{1}$ State Key Laboratory of the Gas Disaster Detecting, Preventing and Emergency Controlling, Chongqing 400037, China \\ ${ }^{2}$ China Coal Technology and Engineering Group Chongqing Research Institute, Chongqing 400037, China \\ ${ }^{3}$ Taiyuan University of Technology, Sch Safety \& Emergency Management Engn, Taiyuan, 030024 Shanxi, China
}

Correspondence should be addressed to Jie Cao; cqcaoj@126.com

Received 6 May 2021; Accepted 8 June 2021; Published 18 June 2021

Academic Editor: Yang Wang

Copyright (c) 2021 Xusheng Zhao et al. This is an open access article distributed under the Creative Commons Attribution License, which permits unrestricted use, distribution, and reproduction in any medium, provided the original work is properly cited.

Coal and gas outburst is still a major safety problem in the process of coal production in China. Correctly understanding of the migration law of outburst high gas and pulverized coal is an important basis for accurately predicting the occurrence time and possible scope of outburst. To reveal the airflow disturbance characteristics and coal-gas flow rule in coal and gas outburst process, outburst coal-gas migration simulations under different gas pressures were conducted using a self-developed visual outburst dynamic effect test device. The results showed that coal-gas flow state at the outburst port is divided into subcritical flow, critical flow, and supercritical flow state. The pulverized coal-gas flow migration in the roadway space can be divided into coal gas two-phase flow area, air compression area, and undisturbed area. Under the experimental conditions, the maximum propagation velocities of wave are $342.22 \sim 359.21 \mathrm{~m} / \mathrm{s}$, and the coal gas two-phase flow is far less than the propagation velocities of outburst wave, just $3.68 \sim 33.33 \mathrm{~m} / \mathrm{s}$. When the outburst energy is large, multiple compression waves can superimpose to form shock waves. The peak value of the wave does not necessarily appear in the first boosting range. The presence of pulverized coal leads to a faster attenuation of shock wave, but it makes a greater dynamic destructive force at the same speed.

\section{Introduction}

Coal has always been China's most important energy source. In 2019 , coal consumption accounted for $57.7 \%$ of the country's total energy consumption [1]. Because of the complex conditions for the occurrence of coal seams, China has always been the country with the most serious coal and gas outburst disasters. Several measures and policies have been enacted to prevent and control outburst disasters, such as shutting down a large number of outburst mines, encouraging the development of new technologies, and issuing new rules for the Prevention and Control of Coal and Gas Outburst. In this context, the number of outburst accidents and deaths in China has shown a downward trend (as shown in Figure 1). Compared with 2012, the number of outburst accidents and deaths in 2019 decreased by $66.7 \%$ and $63.6 \%$, which is close to the reduction in the number of coal mines (62.1\%). In the second half of 2019, three serious coal and gas outburst acci- dents occurred in Guizhou Province, resulting in 27 deaths and exceeding the same period in 2017, which reflected the uncertainty and complexity of outburst control work. Outburst disasters have not been well contained.

In the process of outburst, the adsorbed gas in the coal is desorbed quickly to generate a high-pressure gas flow. And continue to carry broken coal (rock) powder rapidly thrown out of the coal wall, producing a significant dynamic effect in the mining space. The strong compression wave generated by outburst cause casualties and damage roadway facilities. Additionally, the high-concentration gas that is emitted flows along the disturbance area and may even flow back into the air inlet lane, which may induce secondary disasters, such as gas explosions, and cause extensive casualties. For example, on November 21, 2009, after coal and gas outburst occurred in Xinxing Coal Mine in China, gas explosion was induced by gas countercurrent and electric spark, causing secondary damage. A total of 108 people died in the accident, 


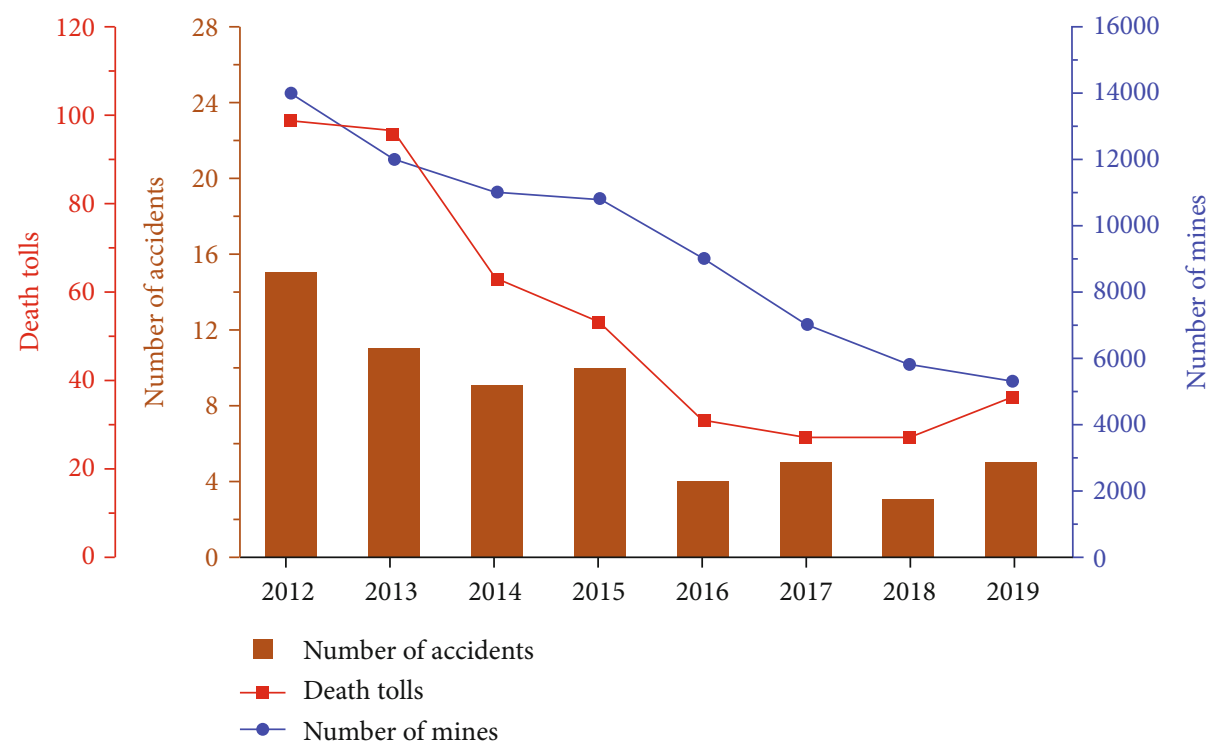

Figure 1: The number of mines, coal and gas outburst accidents, and the death tolls in recent years.

80 of them died of $\mathrm{CO}$ poisoning caused by gas explosion. On March 31, 2010, an extraordinarily serious coal and gas outburst occurred at the Henan National Coal Company, which caused an explosion and combustion of gas out of the wellhead, causing 44 deaths.

Most researches focus on the occurrence conditions of coal and gas outburst [2-11]. Few studies, however, have examined the dynamic hazard effect of high-pressure gaspulverized coal in roadway space in the outburst process. The migration of high-pressure gas and pulverized coal in the roadway space during an outburst is a complicated gassolid two-phase flow problem. This problem has a certain similarity with the horizontal pipeline pneumatic conveying problem. Sheng [12] assumed outburst coal to be a particle group and constructed a mathematical model of outburst coal particle migration. Zhang [13] studied the transportation model of outburst coal and gas in the roadway by drawing on the theory of pneumatic transportation and used numerical simulation methods to study the movement law of coal and gas. The mathematical model of the migration law of coal outburst in roadway in one dimension was established by Sun et al. [14, 15]. The movement process of outburst coal particles was divided into acceleration, equilibrium deceleration, and settlement stages, and the suspension movement mechanism of solid particles in the airflow and the law of conservation of energy was used comprehensively. Zhao et al. [16] analyzed the flow state and transportation mechanism of coal-gas flow during coal and gas outburst.

Otuonye and Sheng [17] were the first to study the dynamic process of the shock airflow formed by the outburst and established an outburst shock wave mathematical model. Fedorov and Fedorchenko [18] analyzed the migration law of coal and gas two-phase flow and identified the relationships among shock wave velocities and initial concentration, solid particle diameter. By analyzing the Zhongliangshan outburst test observation, Cheng et al. [19, 20] believe that the pressure wave generated by the outburst was always positive pressure during the propagation process because of the rapid desorption of gas from pulverized coal. Based on this viewpoint, an outburst shock wave propagation model was constructed. Wang [21] obtained the calculation formula of the reflected overpressure acting on the reverse antioutburst door based on the analysis of the shock wave model and calculated the mine outburst gas counterflow based on the onedimensional gas flow model. Zhang et al. [22] considered that the outburst shock wave was a weak shock wave, and its propagation attenuation was related to such factors as the initial energy of the shock wave, propagation distance, and frictional resistance characteristics of the roadway. A calculation formula for outburst shock wave expressed by overpressure was constructed. Yang and Zhang [23] used gas dynamics theory to establish the relationship between shock wave overpressure, flow velocity and propagation distance, coal seam gas pressure, and other parameters and calculated the range of gas flow propagation damage under different overpressures.

These studies conducted qualitative analysis mostly from a theoretical perspective and lacked quantitative data for support and verification. In recent years, with increasing demand for research on the mechanism of outburst catastrophe, some experimental devices for outburst coal-gas twophase flow simulation with gas as the main power source have been developed, which provide basis for the study of outburst dynamic effects. Based on the principle of segmented pressure difference, an outburst simulation experimental device was developed [24, 25]. Using this device, Wang et al. [26] studied the influence of gas pressure, roadway bifurcation angle, and roadway crosssection on the propagation of outburst shock airflow through experiments and constructed a propagation model of outburst shock airflow in bend roadways, variable crosssection roadways, and bifurcated roadways. Using the working principle of laser schlieren, experimental system for the impact dynamic effect of gas pulverized coal flow was built [27]. It is used to study 
the outburst impact on the obstacles in the roadway. The study found that the kinetic energy of outburst gas pulverized coal flow was converted into pressure potential energy through a time accumulation process, and its impact on the obstacles transitioned from strong to weak until the final cut-off. Jin et al. [28] revealed the phenomenon of selfacceleration of the outburst pulverized coal, which is under the influence of high pressure, and the rapid desorption of initial gas in coal, and described the flow pattern of outburst coal-gas two-phase flow. Sun et al. [29] developed a simulation test device for the dynamic effects of coal and gas outbursts. The outburst hole size of the device was up to 1.88 $\mathrm{m}^{3}$, and the simulated roadway was $50 \mathrm{~m}$ long. The propagation law of the outburst shock wave along the main and bifurcated roadway was analyzed through experiments. The average attenuation coefficient of the outburst shock wave at the connecting part in the main roadway was about five times greater than other places because of the diversion. Through tests, the influence of the particle size of the outburst pulverized coal on the gas impact force was obtained, and the velocity of the gas flow shock wave was much greater than the velocity of the pulverized coal movement found by Wang et al. [30]. Zhou et al. [31, 32] developed a dynamic disaster visual test system based on their multifunctional coal mine gas dynamic disaster simulation experiment device. The characteristics of outburst coal movement can be observed through the observation windows made of polymethyl methacrylate on the roadway. Based on the analysis of coal-gas two-phase flow dynamic behavior during the outburst process, the roadway was divided into five parts: airflow disturbance area, air compression area, coal-gas two-phase flow migration area, shock wave area, and undisturbed area.

Because of different experimental methods and purposes, some differences are evident in the experimental rules. In the observations of some scholars $[29,30,33,34]$, the peak value of the maximum overpressure in the roadway is about 100 $\mathrm{kPa}$, and there is no negative pressure or relatively small negative pressure value. However, in experimental observation of Jin et al. [28], the gas pressure in the roadway presents multiple fluctuations of positive and negative pressure. Presently, there are many studies on the propagation law of the outburst shock wave, but there is no unified understanding of its causes and characteristics. Therefore, a self-developed visual coal and gas outburst dynamic effect test device was used to observe the quantitative law of outburst pulverized coal-gas migration under different initial gas pressure conditions and analyze the characteristics of airflow disturbance in the outburst process, which can provide important support for revealing the mechanism of outburst shock wave and accurately judging the occurrence time and possible range of outburst.

\section{Experiment of Pulverized Coal-Gas Two- Phase Flow Migration in Roadway}

2.1. Experimental System. During the development stage of coal and gas outburst, the broken coal body is thrown out from the outburst hole to the roadway space under the action of high gas pressure flow. To observe the outburst wave and outburst pulverized coal movement characteristics in the roadway during the outburst process, a set of outburst coalgas flow visual simulation experimental device was designed. The device consisted of a high-pressure gas cylinder, outburst chamber, visual simulation pipeline, sensors, and high-speed camera (as shown in Figure 2). The outburst chamber was made of cylindrical stainless steel, the inner cavity diameter was $20 \mathrm{~cm}$, the length was $30 \mathrm{~cm}$, and the designed pressure resistance was $5 \mathrm{MPa}$. To simulate the feature of the outburst hole "small mouth and large cavity," a $10 \mathrm{~cm}$ diameter opening was reserved at the front end to simulate the outburst port, which was controlled by a solenoid valve and connected with a visual simulation pipeline. The visual simulation pipeline was made of acrylic material with high light transmittance and high strength. The inner diameter was also 10 $\mathrm{cm}$. The pipes were connected and sealed with flanges and gaskets. The main parameters of the experimental apparatus are shown in Table 1. The advantage is that the ball solenoid valve can accurately and effectively control the opening of the outburst port, and the transparent visual simulation pipeline with high-speed camera can capture the entire process of outburst coal transportation.

2.2. Experimental Scheme. To explore the migration law of outburst coal-gas two-phase flow in the roadway space, taking carbon dioxide as the experimental gas, the outburst simulation experiment was carried out under different initial gas pressure (0.1 MPa, 0.3 MPa, and $0.5 \mathrm{MPa})$ conditions. Before the experiment, the visual simulation pipeline was connected, and sensors and high-speed cameras were installed. The selected $(3-10 \mathrm{~mm})$ fresh coal sample into the outburst chamber was put, and it was manually tamped with the pressure plate. The coal density after tamping was about $1 \mathrm{~g} / \mathrm{cm}^{3}$. Then, the experimental gas was filled and fully adsorbed. The pneumatic solenoid valve was used to open the outburst port to start the experiment. The data was monitored, and the outburst pulverized coal movement in real time was photographed. After the completion of the experiment, the coal samples that were thrown out in the roadway were collected and statistically screened.

\subsection{Experimental Results}

2.3.1. The Law of Outburst Wave Propagation. The gas pressure variations in the outburst chamber and simulation pipeline with time under different gas pressure conditions are shown in Figure 3. As the outburst progressed, the gas pressure in the chamber gradually decreased, and the pressure at each monitoring point in the simulation pipeline showed a positive pressure and negative pressure pulsating development. When the outburst occurred, a large amount of free gas was sprayed out from outburst hole to disturb the air in the roadway, forming a disturbance wave.

It can be seen from Figure 3 that the pressure decay time in the cavity was different under different test conditions (about 200-350 ms to atmospheric pressure), and it showed a decreasing trend with the decrease of initial gas pressure. When the initial gas pressure was $0.3 \mathrm{MPa}$ and $0.5 \mathrm{MPa}$, as the gas pressure in the chamber decreased, two to three 


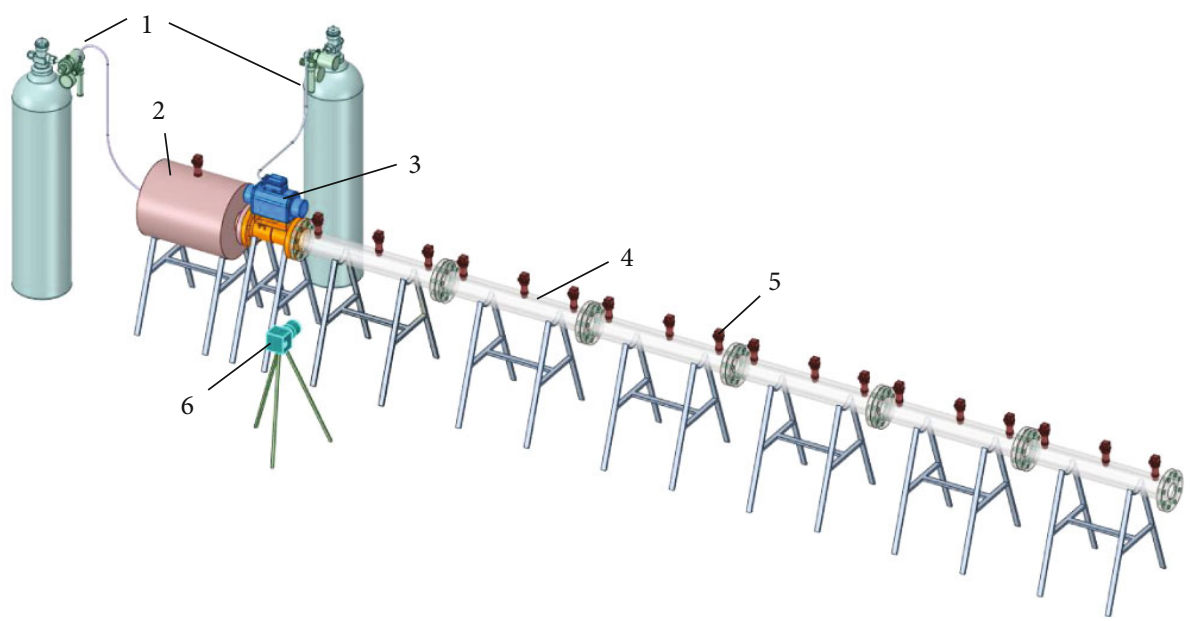

FIgURE 2: The visual simulation experimental device of outburst coal-gas flow. 1. High-pressure gas cylinder; 2 . outburst chamber; 3. pneumatic solenoid valve; 4 . visual simulation pipeline; 5 . sensors; 6 . high-speed camera.

TABLE 1: Main parameters of the device and the existing ones.

\begin{tabular}{lcccc}
\hline Technical parameter & Outburst cavity size/cm & Outburst opening mode & Simulated pipe type & Simulated pipe size/cm \\
\hline & $\varphi 20 \times 30$ & Ball valve & Acrylic material & $\varphi 10$ \\
Chen et al. [24, 25] & $\varphi 30 \times 45$ & Mechanical damper & Metal & $\varphi 20$ \\
Hu [27] & $\varphi 30 \times 45$ & Rupture of membranes & Plexiglass & $20 \times 20$, arch radius 10 \\
Jin [28] & $\varphi 20 \times 30$ & Mechanical damper & Acrylic material & $\varphi 10$ \\
Sun et al. [29] & $\varphi 100 \times 236$ & Rupture disk & Metal, viewing window & $30 \times 30$ \\
Zhou et al. [31, 32] & $105 \times 41 \times 41$ & Rupture disk & Metal, viewing window & $40 \times 40$ \\
\hline
\end{tabular}

consecutive compression waves and expansion waves appeared at different locations in the simulation pipeline. Under the condition of $0.3 \mathrm{MPa}$ (No. 3), the time of the first compression wave occurred between 0 and $50 \mathrm{~ms}$, and the time of the second compression wave occurred between $100 \mathrm{~ms}$ and $165 \mathrm{~ms}$. When the initial gas pressure was 0.1 $\mathrm{MPa}$, the gas pressure fluctuation in the roadway was less than $2 \mathrm{kPa}$, which could be ignored. The peak pressure $\Delta$ $p_{i}$ of the compression wave at different positions of the roadway $(i=1,2,3$, represents the order in which compression wave appear) is shown in Table 2 . It can be seen that the peak of the compression wave basically decayed along the roadway, especially when it was near the end of the roadway and when the attenuation was faster. At the same position in the roadway, as the pressure of the outburst chamber decreased, the peak pressure of the first compression wave $\Delta p_{1}$ was generally the largest, and then, it gradually decreased.

It is assumed that the air velocity in the roadway before the outburst is zero relative to the initial pressure of the outburst flow. At this time, the wave front air pressure is $p_{0}$, and the density is $\rho_{0}$, the temperature is $T_{0}$, the spatial coordinate of wavefront takes the outburst point as the coordinate origin, and the distance is expressed by $L$. The parameters on the wave front are pressure $p_{1}$, density $\rho_{1}$, the velocity of air flow behind the wave is $u_{1}$, and the velocity of wave front is $D$. Then, the relationship between the parameters of the outburst wave expressed by the maximum overpressure $\left(\Delta p_{1}\right)$ is

$$
\left\{\begin{array}{l}
D=\sqrt{c_{0}^{2}+\frac{k+1}{2 \rho_{0}} \Delta p_{1},} \\
u_{1}=\frac{\Delta p_{1}}{\rho_{0} c_{0} \sqrt{1+\left((k+1) /\left(2 \rho_{0} c_{0}^{2}\right)\right) \Delta p_{1}}},
\end{array}\right.
$$

where $k$ is the air compression coefficient, taking $1.4 ; c_{0}$ is the sound velocity under the standard state, taking 340 $\mathrm{m} / \mathrm{s}$. According to Equation (1), the propagation velocity of the outburst wave front and the air flow at different positions in the roadway can be calculated (as shown in Table 3). It can be seen from Table 3 that with the increase of the propagation distance of the outburst wave, the propagation velocity of the outburst wave front and the gas flow shows an attenuation trend. On the one hand, the propagation velocity of outburst wave is positively correlated with overpressure. On the other hand, the propagation speed of the outburst wave depends on the local sound speed. The continuous compression of the gas makes the local sound speed increase, and the absolute speed of the compression wave generated behind must be faster than that in front. The 


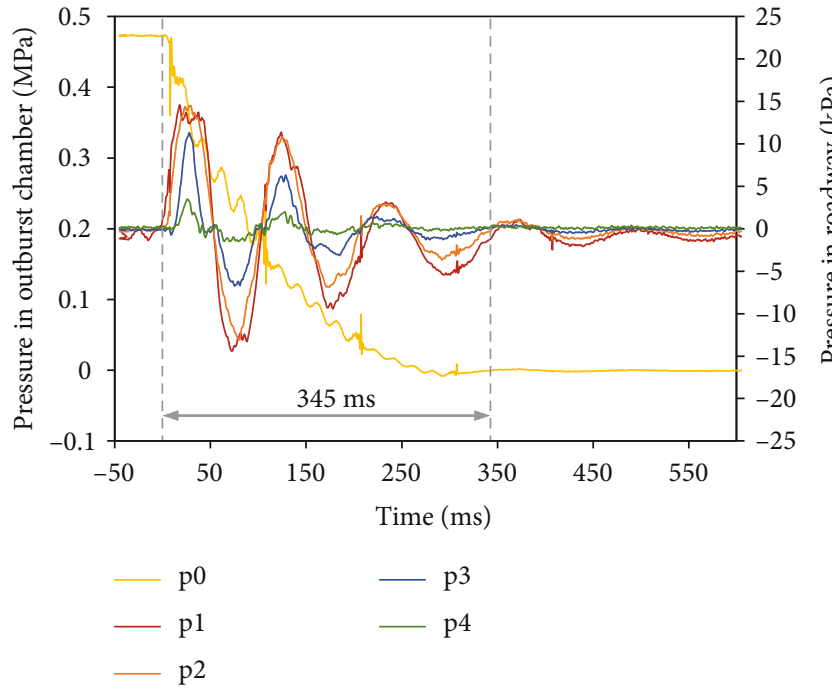

(a)

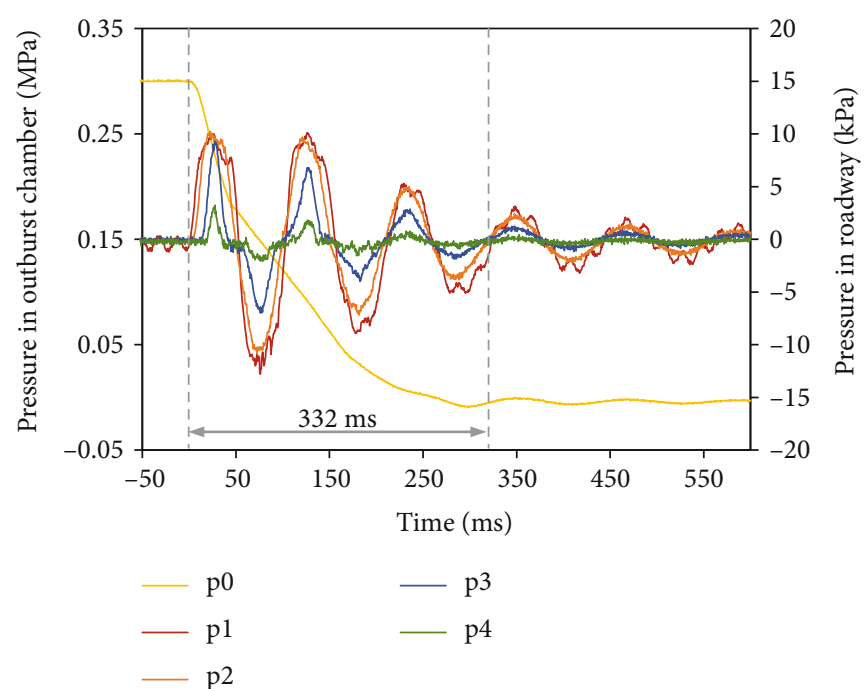

(b)

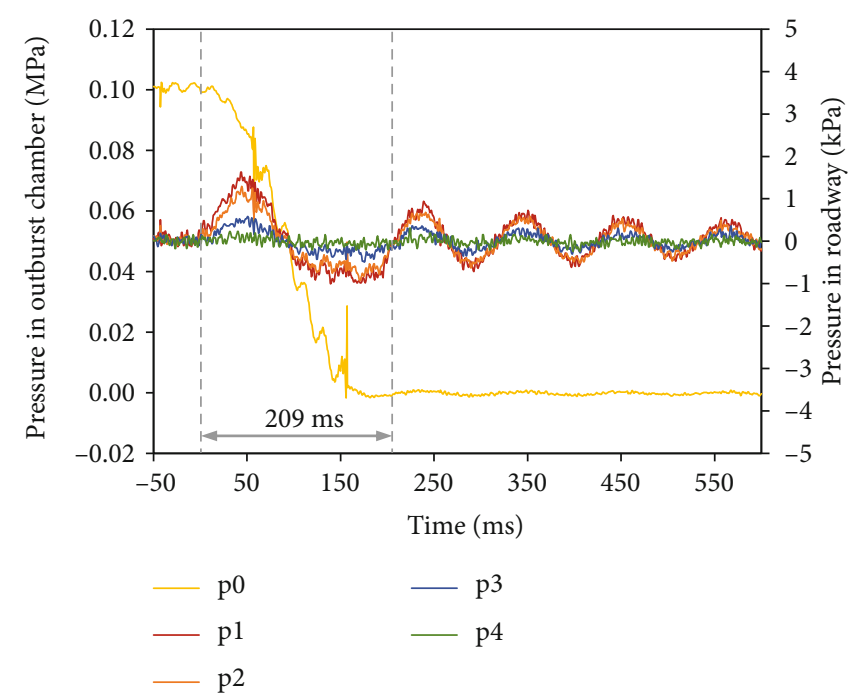

(c)

FIgURE 3: Evolution curve of pressure in outburst chamber and simulation pipeline with time. (a) $0.5 \mathrm{MPa}$; (b) $0.3 \mathrm{MPa}$; (c) $0.1 \mathrm{MPa}$.

TABLE 2: The peak pressure value of compression wave at different positions in the roadway.

\begin{tabular}{|c|c|c|c|c|c|c|c|}
\hline \multirow{3}{*}{ Gas pressure sensor } & \multirow{3}{*}{$\begin{array}{l}\text { Distance from } \\
\text { outburst port/m }\end{array}$} & \multicolumn{6}{|c|}{ Peak pressure of compression wave $/ \mathrm{kPa}$} \\
\hline & & & $0.5 \mathrm{MPa}$ & & & $0.3 \mathrm{MPa}$ & \\
\hline & & $\Delta p_{1}$ & $\Delta p_{2}$ & $\Delta p_{3}$ & $\Delta p_{1}$ & $\Delta p_{2}$ & $\Delta p_{3}$ \\
\hline p1 & 0.5 & 14.595 & 11.395 & 3.125 & 9.99 & 10.09 & 5.49 \\
\hline $\mathrm{p} 2$ & 2.5 & 14.504 & 10.804 & 2.904 & 10.21 & 9.69 & 4.983 \\
\hline p3 & 5.5 & 11.29 & 6.32 & 1.44 & 9.27 & 6.81 & 2.854 \\
\hline $\mathrm{p} 4$ & 7.5 & 3.512 & 1.982 & 0.769 & 3.22 & 1.79 & 0.77 \\
\hline
\end{tabular}

larger the initial gas pressure is, the greater the expansion energy of released gas is, and the faster the propagation speed of outburst wave is.

2.3.2. The Movement Process of Outburst Coal-Gas in the Roadway. The flow pattern of the outburst coal-gas twophase flow uses a high-speed camera. According to the theory of pneumatic conveying, following are the types of flow states of outburst coal-gas two-phase flow: suspension flow, dune flow, stratified flow, and slug flow [16]. It is considered that outburst pressure, coal particle size distribution, and ejection distance significantly affect the flow pattern [28]. The movement process of outburst coal and gas in the first roadway section under the $0.3 \mathrm{MPa}$ initial gas pressure condition is 
TABLE 3: The propagation speed of outburst wave under different initial gas pressure.

\begin{tabular}{|c|c|c|c|c|c|c|}
\hline $\begin{array}{l}\text { Distance from } \\
\text { outburst port } / \mathrm{m}\end{array}$ & $0.5 \mathrm{MPa}$ & $\begin{array}{c}u_{1} / \mathrm{m} / \mathrm{s} \\
0.3 \mathrm{MPa}\end{array}$ & $0.1 \mathrm{MPa}$ & $0.5 \mathrm{MPa}$ & $\begin{array}{c}D / \mathrm{m} / \mathrm{s} \\
0.3 \mathrm{MPa}\end{array}$ & $0.1 \mathrm{MPa}$ \\
\hline 0.5 & 31.15 & 20.72 & 3.68 & 359.20 & 352.66 & 342.22 \\
\hline 2.5 & 31.15 & 19.00 & 2.63 & 359.21 & 351.59 & 341.58 \\
\hline 5.5 & 26.57 & 14.98 & 1.32 & 356.31 & 349.10 & 340.79 \\
\hline 7.5 & 5.83 & 2.92 & 0.61 & 343.52 & 341.76 & 340.37 \\
\hline
\end{tabular}

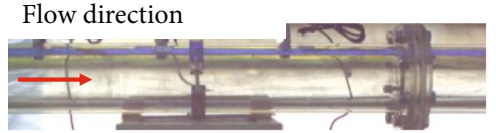

(a)

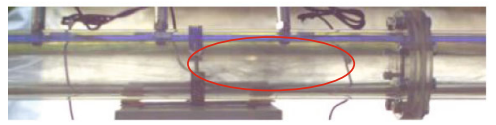

(b)

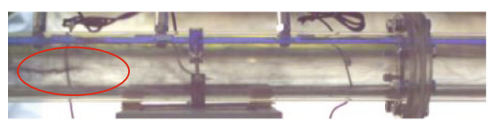

(c)

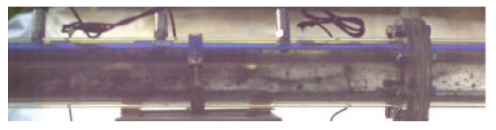

(d)

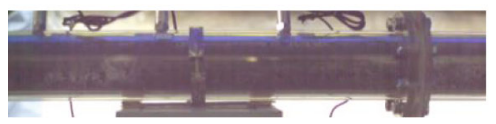

(e)

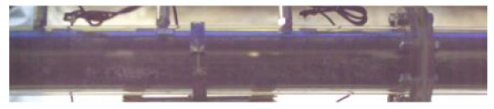

(f)

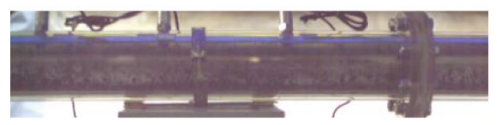

$(\mathrm{g})$

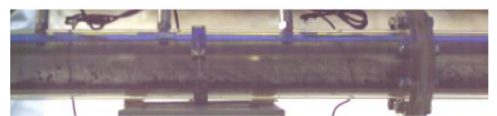

(h)

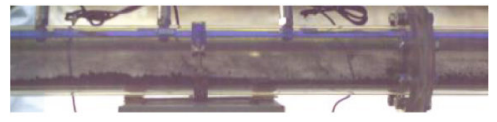

(i)

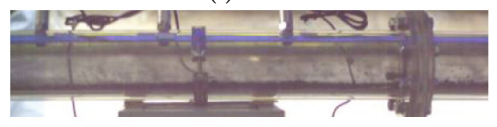

(j)

FIGURE 4: The evolution process of two-phase flow in the first section during outburst (0.3 MPa). (a) $0 \mathrm{~ms}$; (b) $50 \mathrm{~ms}$; (c) $110 \mathrm{~ms}$; (d) $250 \mathrm{~ms}$; (e) $400 \mathrm{~ms}$; (f) $500 \mathrm{~ms}$; (j) $600 \mathrm{~ms}$; (h) $800 \mathrm{~ms}$; (i) $1000 \mathrm{~ms}$; (j) $1400 \mathrm{~ms}$.

shown in Figure 4. The sampling frequency of the highspeed camera was 500 frames. Figure 4(a) shows the initial state when the outburst had not yet occurred. It can be seen from Figure 4 that when the outburst was just excited $(<110$ $\mathrm{ms})$, gas flow was dominant. Under the action of the highspeed gas flow, the fine pulverized coal particles were carried into the roadway, and the flow was in a dilute-phase suspension flow state (Figure 4(b)). At $110 \mathrm{~ms}$ (Figure 4(c)), some of the pulverized coal particles moved forward at the center of the pipeline. From this point on, the amount and particle size of pulverized coal particles carried by the emitted gas increased, and the suspended flow density began to increase, which gradually showed a continuous dense phase flow state. At $250 \mathrm{~ms}$ (Figure 4(d)), the entire roadway space was filled; the amount of pulverized coal particles increased further (Figure 4(e)) and reached the maximum at $500 \mathrm{~ms}$ (Figure 4(f)). Subsequently, the large particles of pulverized coal settled to present a dune flow (Figures 4(g) and 4(h)) and gradually evolved into a laminar flow (Figures 4(i) and $4(j))$ until the movement ceased. The movement state of the two-phase flow in the outburst process was not stable. Under the experimental conditions, the outburst coal-gas flow experienced roughly four stages: a gas-based dilute-phase suspen- sion flow state, a change to a continuous dense phase flow state, and then, a change to dune flow and laminar flow. The entire migration time of pulverized coal particles was longer than $1400 \mathrm{~ms}$, and the gas-solid parameters also changed with time because of changes in the motion state. Compared with Figure 3(b), it is evident that in the first compression wave stage, only a very small amount of pulverized coal flowed under the action of the gas flow in the first section of pipeline. A certain amount of pulverized coal was thrown out at the beginning of the second compression wave. Even after pressure reduced to atmospheric pressure, a large amount of pulverized coal still moved in the roadway space. This verified that the outburst coal migration in the roadway lagged behind the airflow disturbance in the roadway.

The average velocity of pulverized coal flow in different positions of roadway during outburst can be obtained by image measurement method (as shown in Figure 5). It can be seen that under the gas pressure of $0.5 \mathrm{MPa}, 0.3 \mathrm{MPa}$, and $0.1 \mathrm{MPa}$, the corresponding maximum pulverized coal flow velocities are $33.33 \mathrm{~m} / \mathrm{s}, 13.88 \mathrm{~m} / \mathrm{s}$, and $5 \mathrm{~m} / \mathrm{s}$, respectively, which are close to the propagation velocity of gas flow. The greater the gas pressure is, the greater the gas pressure gradient between the cavity and the simulated roadway space 


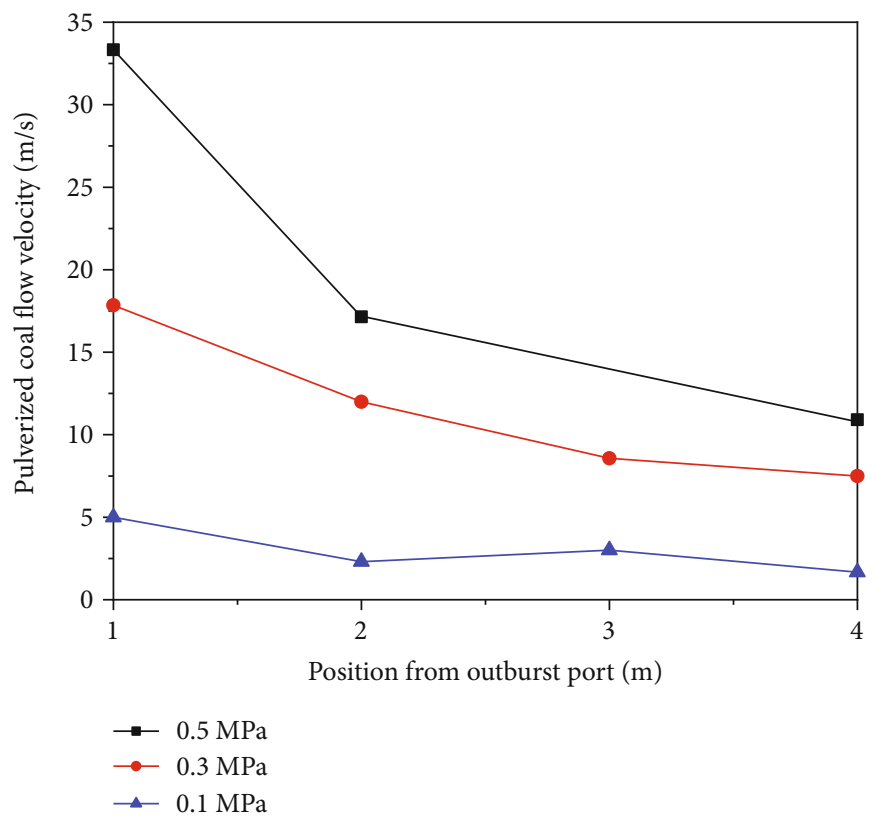

FIGURE 5: Relationship between pulverized coal flow velocity and position in outburst process.

is, and the greater the coal gas two-phase flow velocity is. With the increase of two-phase flow movement distance, gas pressure and gas flow velocity decrease gradually, and the ability of carrying or pushing pulverized coal decreases gradually. In addition, with the increase of roadway distance, the migration velocity of pulverized coal flow decreases.

2.3.3. Coal Powder Distribution and Outburst Energy. The outburst strength (outburst coal quality) was different under different conditions. The mass of outburst coal under the test initial gas pressure conditions of $0.5 \mathrm{MPa}, 0.3 \mathrm{MPa}$, and 0.1 MPa was $1.0486 \mathrm{~kg}, 0.8523 \mathrm{~kg}$, and $0.4749 \mathrm{~kg}$, respectively. The occurrence and development of outburst is the result of energy accumulation and dissipation. When the elastic energy and gas internal energy stored in the outburst coal seam reach a certain level, an outburst is possible $[35,36]$. Ignoring frictional heat, vibration, and sound in the process of outburst, the energy involved in the outburst is transformed into coal transport energy and coal crushing energy. According to statistical analysis, the outburst elastic energy is two to three orders of magnitude smaller than the gas internal energy, and the gas internal energy accounts for more than $98 \%$ of the total energy. Then, the outburst energy formula [37] can be expressed as follows:

$$
\left\{\begin{array}{l}
W_{2}=A_{1}+A_{2}, \\
A_{1}=\frac{M_{t} g L_{p}^{2}}{2 h}, \\
A_{2}=\alpha S=\alpha \frac{6}{\rho}\left(\frac{1}{d}-\frac{1}{D}\right),
\end{array}\right.
$$

where $W_{2}$ is the gas internal energy, J; $A_{1}$ is the coal transport energy, J; $A_{2}$ is the coal crushing energy, J; $M_{\mathrm{t}}$ is the amount of outburst coal, $\mathrm{kg} ; g$ is the gravitational coefficient, 9.8
$\mathrm{N} / \mathrm{kg} ; L_{\mathrm{g}}$ is the equivalent distance of the outburst coal powder, $\mathrm{m} ; h$ is the inside diameter of the simulated pipeline, $\mathrm{m} ; \alpha$ is the crushing specific work, $\mathrm{J} / \mathrm{m}^{2} ; S$ is the newly added surface area produced by coal sample after crushing, $\mathrm{m}^{2} / \mathrm{kg} ; \rho$ is the apparent density of coal after crushing, $\mathrm{kg} / \mathrm{m}^{3}$; and $d$ and $D$ are the average diameters of the coal after and before the outburst, $\mathrm{m}$. By calculation, the total energy involved in the outburst of $0.5 \mathrm{MPa}, 0.3 \mathrm{MPa}$, and $0.1 \mathrm{MPa}$ initial gas pressure experiments was $1815.16 \mathrm{~J}, 1060.01 \mathrm{~J}$, and $212.91 \mathrm{~J}$, respectively. This showed that the greater the gas pressure, the greater the outburst strength and energy.

\section{Discussion}

3.1. Formation Conditions of Outburst Shock Wave. From the experimental results, under $0.3 \mathrm{MPa}$ and $0.5 \mathrm{MPa}$ gas pressure conditions, it was evident that multiple compression waves and expansion waves were generated in the roadway after the outburst, but this may have been caused by the fact that the total energy involved in the outburst was small (the maximum is $1.82 \mathrm{KJ}$ ), and the compression waves were not superimposed to form a shock wave.

The outburst shock wave was generated, however, when the coal and gas outburst dynamic simulation test device was used to conduct the outburst coal-gas flow simulation experiment under the condition of $0.35 \mathrm{MPa}$ [29]. The size of the outburst cavity of the device was larger, $1.88 \mathrm{~m}^{3}$. Using $\mathrm{CO}_{2}$ as the test gas, the mass of outburst pulverized coal was $180.7 \mathrm{~kg}$, and the total energy involved in the outburst was $662 \mathrm{~kJ}$. The gas pressure changes during the outburst simulation experiment are shown in Figure 6. Where $p 0$ represents the gas pressure in the outburst hole, $p 1, p 6$, and $p 10$ represent the gas pressure at $1.5 \mathrm{~m}, 10.25 \mathrm{~m}$, and $22.5 \mathrm{~m}$ from the outburst port in the roadway, respectively. Figure 6 shows that the gas pressure in the outburst hole basically decayed 

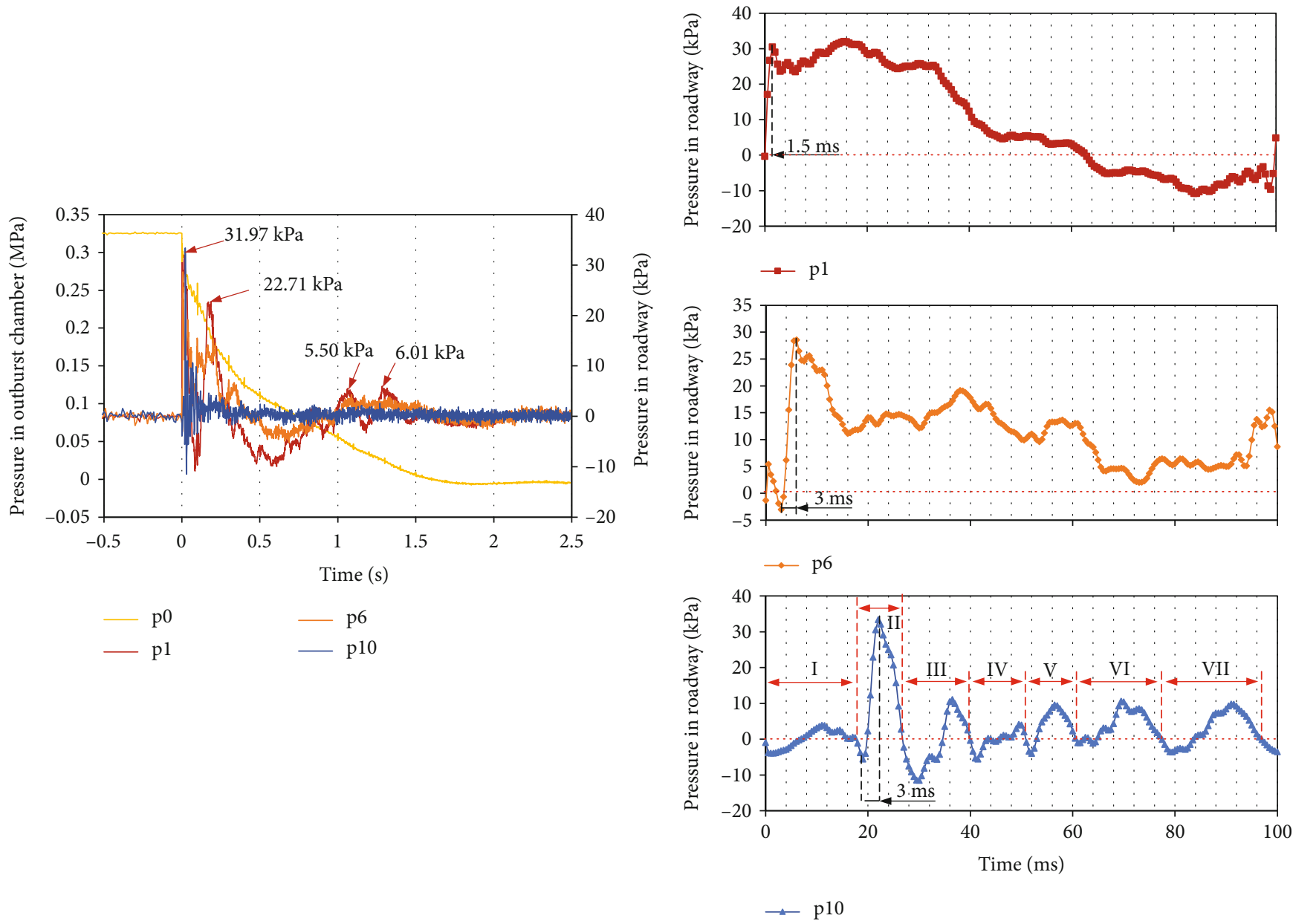

(a)

(b)

Figure 6: Results of large-size outburst coal-gas flow simulation test. (a) Curves of gas pressure of the whole outburst process; (b) curve of pressure in roadway in the first $100 \mathrm{~ms}$ of outburst.

exponentially, lasting $1.6 \mathrm{~s}$ in total. As the gas jet into the roadway space, compression waves and expansion waves alternately appeared. The $p 1$ produced four obvious compression wave peaks in the whole outburst process. With the decrease of gas pressure in the outburst chamber, the peak value of compression wave basically decreased. The third and fourth compression wave peak values of $5.50 \mathrm{kPa}$ and $6.01 \mathrm{kPa}$ were close to the data of $\Delta p 3$ given in Table 2. In the initial stage of the outburst (first $100 \mathrm{~ms}$ ), the $p 1, p 6$, and $p 10$ increased in a very short period of $1.5 \mathrm{~ms}, 3 \mathrm{~ms}$, and $3 \mathrm{~ms}$ (as shown in Figure 6(b)). Meanwhile, the gas pressures of $p 1$ and $p 6$ retained positive pressure within this 100 $\mathrm{ms}$, and $p 10$ showed that expansion wave, and compression wave appeared alternately seven times. It is believed that the outburst energy remained large near the outburst port and that the later compressional wave could catch up with the previous one in this place. As a result, the shock wave was formed by the superposition of multiple compression waves. Because of the energy dissipation in the outburst pulverized coal transportation, the outburst energy far away from the outburst was relatively small, and the propagation rate of compression wave was attenuated, so the shock wave

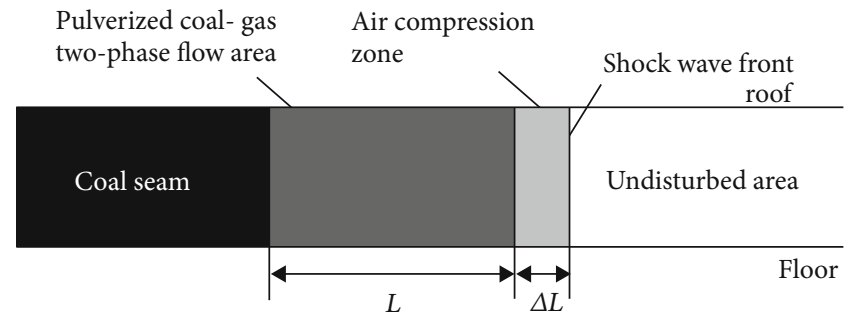

FIGURE 7: The model of outburst wave in the roadway.

could not be superimposed. Therefore, when the gas pressure condition was satisfied, the outburst energy had to reach a certain value to form the outburst shock wave.

3.2. Outburst Wave Characteristics. According to the above analysis and the experimental conditions, the model of outburst wave in the roadway can be established (as shown in Figure 7). After the pressure relief of the high-pressure outburst chamber, the pulverized coal in the outburst chamber flows into the roadway in the form of two-phase flow of pulverized coal and gas under the action of high-pressure gas, 
and the pulverized coal-gas two-phase flow compresses the air within the range of $L$ from the outburst to the maximum pressure $\Delta L$ range. According to the theory of gas dynamics, when the Mach number of the shock wave is 2, the thickness of the shock wave is about $0.00025 \mathrm{~mm}$, which is only twice the average free path of the molecule [19]. Therefore, it can be regarded as a vertical mathematical line $\Delta L$ tends to be positive infinitesimal. The roadway space can be divided into three areas: coal gas two-phase flow area, air compression area, and undisturbed area. The interface of undisturbed area in the air compression area is shock wave front. Under the experimental conditions, the maximum propagation velocities of wave and the coal gas two-phase flow are $359.21 \mathrm{~m} / \mathrm{s}$ and $33.33 \mathrm{~m} / \mathrm{s}$.

Coal and gas outburst is a continuous process, usually lasting from a few seconds to tens of seconds. The gas pressure in the outburst hole during the whole process is not constant, exhibits pulse characteristics, and generally shows an exponential decay trend $[31,38]$. Therefore, the entire process is accompanied by the formation and attenuation of shock waves, which last longer than a typical underground gas or coal dust explosion process. Among the recorded outburst instances, many have data records with multiple loud noises, which are the result of multiple fluid shocks that occur during the outburst process. As shown in Table 2, because of the possible multiple excitation phenomenon, the peak value of the compression wave did not necessarily appear in the first boost range, and the second compression wave peak value was greater than the first compression wave peak value of $p_{1}$ in the $0.3 \mathrm{MPa}$ gas pressure experiment.

The coal and gas outburst process is accompanied by large amount of pulverized coal migration and accumulation, which make the formation and propagation of the later outburst shock wave in the roadway environment contain a high concentration of coal dust. The particle volume concentration of pulverized coal is significantly higher than other explosion shock waves in coal mine. The concentration of pulverized coal particles has a significant influence on the propagation characteristics of the outburst shock wave. As the particles interact with the shock wave, they will absorb a lot of energy, thereby speeding up the attenuation of the shock wave, reducing the destructive effect of the shock wave overpressure, and causing the shock wave propagation distance to be affected. As shown in Figure 6, at 22.5 $\mathrm{m}$ from the outburst port, there was only one large disturbance wave in the area, which was significantly different from the change in gas pressure near the outburst port. At the same time, the presence of pulverized coal increased the quality of the high-speed moving air flow behind the wave, which increased the dynamic pressure destructive force at the same moving speed. Wang et al. [30] conducted experimental research and found that the outburst pulverized coal strike force was up to $1.4 \mathrm{MPa}$, which was about 35 times that of the gas peak pressure.

\section{Conclusions}

To reveal the airflow disturbance characteristics and coal-gas flow rule in coal and gas outburst process, outburst coal-gas migration simulations under different gas pressures were conducted using a self-developed visual outburst dynamic effect test device; the following conclusions can be drawn:

(1) The outburst coal-gas flow experienced four stages and the migration of outburst coal lagged behind the initial airflow disturbance in the roadway space. Under the experimental conditions, the maximum propagation velocities of wave are 342.22 359.21 $\mathrm{m} / \mathrm{s}$, and the coal gas two-phase flow is far less than the propagation velocities of outburst wave, just $3.68 \sim 33.33 \mathrm{~m} / \mathrm{s}$

(2) When the outburst energy was large, the latter compression wave caught up with the previous one, and multiple compression waves in the roadway superimposed to form a shock wave. The gas pressure in the roadway near the outburst port increased in a very short time and lasted for a period of time in the initial stage of outburst (the first $100 \mathrm{~ms}$ )

(3) The peak value of the outburst disturbance wave generated by the outburst did not necessarily appear in the first boosting range. The outburst coal particles accelerated the attenuation speed of the shock wave. The presence of pulverized coal increased the mass of high-speed air flow after the shock wave, which made its dynamic pressure destructive force increase under the same movement speed

\section{Data Availability}

The data used to support the findings of this study are included within the article.

\section{Conflicts of Interest}

The authors declare that they have no conflicts of interest.

\section{Acknowledgments}

This study was financially supported by the National Natural Science Foundation of China (51774319, 51974358, and 51874348) and Natural Science Foundation of Chongqing, China (cstc2019jcyj-msxmX0531).

\section{References}

[1] National Bureau of statistics, Statistical Bulletin of National Economic and Social Development of the People's Republic of China in 2019, 2020, //http://www.stats.gov.cn/tjsj/zxfb/ 202002/t20200228_1728913.html.

[2] A. A. Skochinski, "Communication of the initiation of a sudden outburst of gas and coal in the model in outburst laboratory of the Institute of Mining of AN SSSR," Ugol, vol. 10, 1953.

[3] A. D. Alexeev, V. N. Revva, N. A. Alyshev, and D. M. Zhitlyonok, "True triaxial loading apparatus and its application to coal outburst prediction," International Journal of Coal Geology, vol. 58, no. 4, pp. 245-250, 2004. 
[4] J. Sobczyk, "A comparison of the influence of adsorbed gases on gas stresses leading to coal and gas outburst," Fuel, vol. 115, no. 2, pp. 288-294, 2014.

[5] Q. Y. Tu, Y. P. Cheng, P. K. Guo, J. Y. Jiang, L. Wang, and R. Zhang, "Experimental study of coal and gas outbursts related to gas-enriched areas," Rock Mechanics and Rock Engineering, vol. 49, no. 9, pp. 3769-3781, 2016.

[6] G. Z. Yin, C. B. Jiang, J. G. Wang, J. Xu, D. M. Zhang, and G. Huang, "A new experimental apparatus for coal and gas outburst simulation," Rock Mechanics and Rock Engineering, vol. 49, no. 5, pp. 2005-2013, 2016.

[7] L. Yuan, "Control of coal and gas outbursts in Huainan mines in China: a review," Journal of Rock Mechanics and Geotechnical Engineering, vol. 8, no. 4, pp. 559-567, 2016.

[8] D. J. Black, "Review of current method to determine outburst threshold limits in Australian underground coal mines," International Journal of Mining Science and Technology, vol. 29, no. 6, pp. 859-865, 2019.

[9] J. Cao, H. T. Sun, B. Wang et al., "A novel large-scale threedimensional apparatus to study mechanisms of coal and gas outburst," International Journal of Rock Mechanics and Mining Sciences, vol. 118, pp. 52-62, 2019.

[10] B. S. Nie, Y. K. Ma, S. T. Hu, and J. Q. Meng, "Laboratory study phenomenon of coal and gas outburst based on a mid-scale simulation system," Scientific Reports, vol. 9, no. 1, p. 15005, 2019.

[11] C. L. Zhang, E. Y. Wang, J. Xu, and S. J. Peng, "Research on temperature variation during coal and gas outbursts: Implications for outburst prediction in coal mines," Sensors, vol. 20, no. 19, 2020.

[12] J. P. Sheng, Real-Time Simulation of an Outburst and the Flow of Resulting Contaminants in an Underground Coal Mine, Michigan Technological University, USA, 1989.

[13] Y. M. Zhang, Motion Pattern of Gas-Solid Two-Phase Flow in Roadway after Coal and Gas Outburst, Shangdong University of Science and Technology, Qingdao, 2008.

[14] D. L. Sun, Study on the Motion Characteristics of Two Phase Flow and the Mechanism of Dynamic Effects in the Process of Coal-Gas Outburst, Shangdong University of Science and Technology, Qingdao, 2013.

[15] D. L. Sun, J. Cao, F. T. Miao et al., "Migration law of outburst coal and gas in roadway," Journal of China Coal Society, vol. 43, no. 10, pp. 2773-2779, 2018.

[16] W. Zhao, Y. P. Cheng, P. K. Guo, K. Jin, Q. Y. Tu, and H. F. Wang, "An analysis of the gas-solid plug flow formation: new insights into the coal failure process during coal and gas outbursts," Powder Technology, vol. 305, pp. 3947, 2017.

[17] F. Otuonye and J. P. Sheng, "A numerical simulation of gas flow during coal/gas outbursts," Geotechnical and Geological Engineering, vol. 12, no. 1, pp. 15-34, 1994.

[18] A. V. Fedorov and I. A. Fedorchenko, "Numerical modeling of the coal-and-gas outburst gasdynamics," Journal of Mining Science, vol. 46, no. 5, pp. 473-484, 2010.

[19] W. Y. Cheng and G. X. Chen, "Formation and modeling of coal and gas outburst shock wave," Safety in Coal Mine, vol. 31, no. 9, pp. 23-25, 2000.

[20] W. Y. Cheng, X. Y. Liu, K. J. Wang, and X. Li, "Study on regulation about shock-wave-front propagating for coal and gas outburst," Journal of China Coal Society, vol. 29, no. 1, pp. 57-60, 2004.
[21] G. Wang, Catastrophic Damage and Failure Characteristics after Coal and Gas Outburst, Shangdong University of Science and Technology, Qingdao, 2008.

[22] J. F. Zhang, K. Wang, and C. P. Wei, "Formation and propagation of shock waves during coal and gas outbursts," Journal of Mining \& Safety Engineering, vol. 27, no. 1, pp. 67-71, 2010.

[23] S. Z. Yang and R. L. Zhang, "Research on injuries due to shock wave and gas flow from coal and gas outburst," China Safety Science Journal, vol. 22, no. 11, pp. 62-66, 2012.

[24] Y. C. Chen, Experimental Study on Shock Wave Propagation Law of Coal and Gas Outburst, Henan Polytechnic University, Jiaozuo, 2009.

[25] J. P. Wei, H. Q. Zhu, Z. H. Wen, and S. J. Pan, "Coal and gas outburst shock wave propagation experiments," Coal, vol. 19, no. 8, pp. 11-13, 2010.

[26] K. Wang, A. T. Zhou, J. F. Zhang, and P. Zhang, "Real-time numerical simulations and experimental research for the propagation characteristics of shock waves and gas flow during coal and gas outburst," Safety Science, vol. 50, no. 4, pp. 835-841, 2012.

[27] W. X. Hu, Theoretical and Experimental Research for GasPulverized Coal Impactive Dynamic Effect after Outburst, China University of Mining and Technology-Beijing, Beijing, 2013.

[28] K. Jin, Y. P. Cheng, T. Ren et al., "Experimental investigation on the formation and transport mechanism of outburst coalgas flow: implications for the role of gas desorption in the development stage of outburst," International Journal of Coal Geology, vol. 194, pp. 45-58, 2018.

[29] H. T. Sun, J. Cao, M. H. Li et al., "Experimental research on the impactive dynamic effect of gas-pulverized coal of coal and gas outburst," Energies, vol. 11, 2018.

[30] K. Wang, L. Wang, F. Du et al., "Influence of coal powder particle sizes on dynamic characteristics of coal and gas outburst," Journal of China Coal Society, vol. 44, no. 5, pp. 1369-1377, 2019.

[31] B. Zhou, J. Xu, S. J. Peng, J. B. Geng, and F. Z. Yan, “Test system for the visualization of dynamic disasters and its application to coal and gas outburst," International Journal of Rock Mechanics and Mining Sciences, vol. 122, p. 104083, 2019.

[32] B. Zhou, J. Xu, F. Z. Yan et al., "Effects of gas pressure on dynamic response of two-phase flow for coal-gas outburst," Powder Technology, vol. 377, pp. 55-69, 2021.

[33] A. T. Zhou, K. Wang, and Z. Q. Wu, "Propagation law of shock waves and gas flow in cross roadway caused by coal and gas outburst," International Journal of Mining Science and Technology, vol. 24, no. 1, pp. 23-29, 2014.

[34] A. T. Zhou, K. Wang, T. F. Feng, J. W. Wang, and W. Zhao, "Effects of fast-desorbed gas on the propagation characteristics of outburst shock waves and gas flows in underground roadways," Process Safety and Environmental Protection, vol. 119, pp. 295-303, 2018.

[35] W. Zhao, Y. P. Cheng, H. N. Jiang, K. Jin, H. F. Wang, and L. Wang, "Role of the rapid gas desorption of coal powders in the development stage of outbursts," Journal of Natural Gas Science and Engineering, vol. 28, pp. 491-501, 2016.

[36] J. Cao, L. C. Dai, H. T. Sun et al., "Experimental study of the impact of gas adsorption on coal and gas outburst dynamic effects," Process Safety and Environmental Protection, vol. 128, pp. 158-166, 2019. 
[37] G. Wang, M. M. Wu, W. M. Cheng, J. H. Chen, and W. Z. Du, "Analysis of energy conditions for coal and gas outburst and factors influencing outburst intensity," Rock \& Soil Mechanics, vol. 36, no. 10, pp. 2974-2982, 2015.

[38] J. Xu, J. B. Geng, S. J. Peng, M. Yuan, C. L. Zhang, and X. H. Luo, "Analysis of the pulsating development process of coal and gas outburst," Journal of China University of Mining and Technology, vol. 47, no. 1, pp. 145-154, 2018. 